\title{
Costal cartilage ensures low degradation of DNA needed for genetic identification of human remains retrieved at different decomposition stages and different postmortem intervals
}

Abstract

\begin{abstract}
Marcin Tomsia¹, Kornelia Droździok ${ }^{1}$ Gulnaz T. Javan², Rafał Skowronek¹, Michał Szczepański', Elżbieta Chełmecka ${ }^{3}$
\end{abstract}
${ }^{1}$ Department of Forensic Medicine and Forensic Toxicology, Faculty of Medical Sciences in Katowice, Medical University of Silesia in Katowice Poland

${ }^{2}$ Department of Physical Sciences and Forensic Science Programs, Alabama State University, Montgomery, AL, 36104, USA

${ }^{3}$ Department of Statistics, Department of Instrumental Analysis, Faculty of Pharmaceutical Sciences in Sosnowiec Medical University of Silesia in Katowice, Poland

Introduction. The study aimed to evaluate if costal cartilage is a good source of DNA for genetic individual identification tests performed in forensic autopsies.

Materials and Methods. The study included samples of costal cartilage collected from 80 cadavers retrieved from different environments: indoors (flat/hospital), outdoors (primarily in the forest), a coal mine, a fire site, uninhabited buildings, a basement, bodies of fresh water, exhumation sites, and unknown locations. After isolation of DNA chondrocytes, T. Large autosomal chromosome (214 bp), T. Small autosomal chromosome (80 bp), and the Y chromosome (75 bp; for male cadavers), sequences were amplified using real-time PCR. Additionally, 23 autosomal short tandem repeat (STR) loci and $16 \mathrm{Y}$ chromosome STR loci were amplified using multiplex PCR. Forensic DNA typing was done using capillary electrophoresis and all results were analyzed.

Results. There was no statistically significant difference in DNA concentration after T. Large, T. Small autosomal chromosome and the $Y$ chromosome amplification between samples collected from cadavers retrieved from different environments. The DNA degradation index was the same regardless of the postmortem interval. The results show that it is possible to generate a full genetic profile from costal cartilage samples collected from cadavers retrieved from different environments and at different times elapsed after death.

Conclusions. The results suggest that costal cartilage can be routinely collected during forensic autopsies, especially from cadavers at the advanced decomposition stage.

\section{INTRODUCTION}

Cartilage is a type of connective tissue formed by chondrocytes located in an amorphous, extracellular matrix that is rich in collagen and elastic fibers [1]. It is assumed that this matrix may constitute the physical barrier protecting chondrocyte DNA from degradation, including posthumous degradation [2]. The costal cartilage can be useful in toxicity studies [3], in postmortem interval (PMI) estimations [4], in age predictions [5], and forensic genetics [6].

Hard tissues, such as teeth [7], petrous bones [8], or femoral bones [9] are used routinely for identification tests in cases of advanced decomposition [10]. However, DNA isolation from hard tissues is not only time- and laborintensive [11] but also ineffective [12]. For example, bone powder incubation may last up to twenty-four hours [13]; osseous material of different degradation degrees may require different isolation techniques [14]; and yield of DNA isolated from hard tissues is minute $[12,15]$.

In this research, we aimed to show that costal cartilage is a suitable biological material for genetic individual identification tests in cases of advanced cadaver decomposition, regardless of the environmental factors and time elapsed since death that typically affect this process. 


\section{METHODS AND METHODS}

\section{Material Origin and Samples Collection}

All research materials, 71 samples from male and 9 from female cadavers, were collected with the prosecutor's consent during forensic visual inspections to perform individual identification tests or during medicolegal autopsies. Costal cartilage fragments, sized approximately $5 \times 6 \mathrm{~cm}$, were collected from the cadavers' rib arches as shown in detail in Figure 1.

The samples were collected from cadavers found in the following locales: in a flat $(n=16)$; outdoors, primarily in the forest $(n=24)$; in a coal mine $(n=9)$; on a fire site $(n=6)$; in

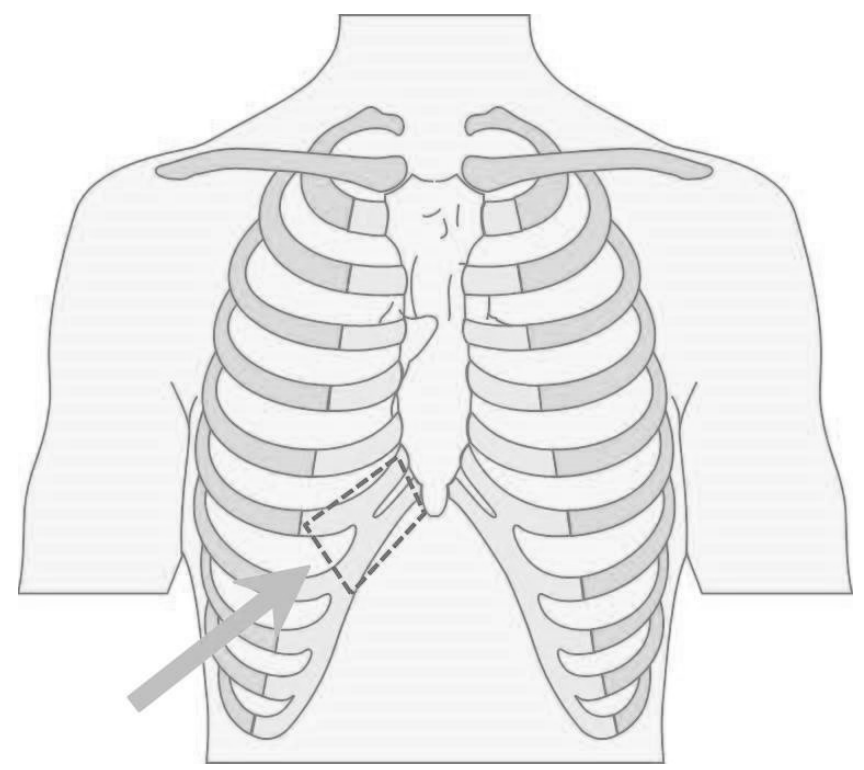

Figure 1. Anatomical location of costal cartilage in the rib cage (light gray color). The arrow shows the place from which the research material was collected

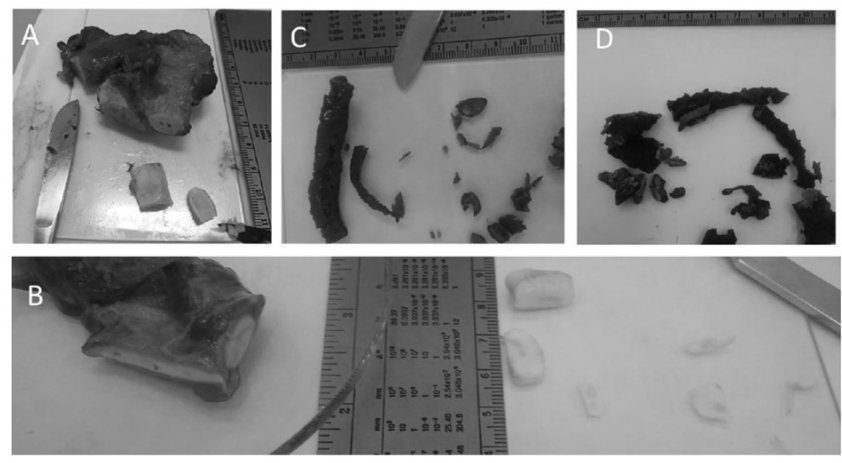

Figure 2. Morphological changes in costal cartilage at different times elapsed since death and at different exposure times to environmental factors: A - few days after death; B - few weeks after death; C - 7 months after death; D - 2 years and 11 months after death an uninhabited building ( $n=4)$; in a basement $(n=3)$; and in a body of fresh water $(n=4)$. Two samples were collected from exhumed bodies and a further three samples from patients after blood transfusions who died in a hospital. Nine samples were collected from cadavers from an unknown location and for those cases, it was impossible to determine factors which could have affected DNA degradation. Whenever possible, the time, the circumstances of death, and the cadaver location were studied. Figures 2 and 3 show morphological changes in the cartilage that occurred and that depended on the time elapsed since death (Figure 2) and on environmental conditions (Figure 3).

\section{Sample Preparation}

Before starting the genetic analysis, the sampled material was cleaned by removing its external surface and any contamination and was then fragmented into cubes using a sterile scalpel blade. Then $80 \mathrm{mg}$ of fragmented cartilage was placed in $1.5 \mathrm{ml}$ Eppendorf tubes and incubated in an extraction mixture (300 $\mu$ l ultrapure $\mathrm{H}_{2}$ Oology, $300 \mu$ lysis buffer, and $20 \mu$ l proteinase $\mathrm{K}$ (Sherlock AX kit, A\&A Biotechn Poland) at $50^{\circ} \mathrm{C}$ and vortexed in a thermomixer at $400 \mathrm{rpm}$

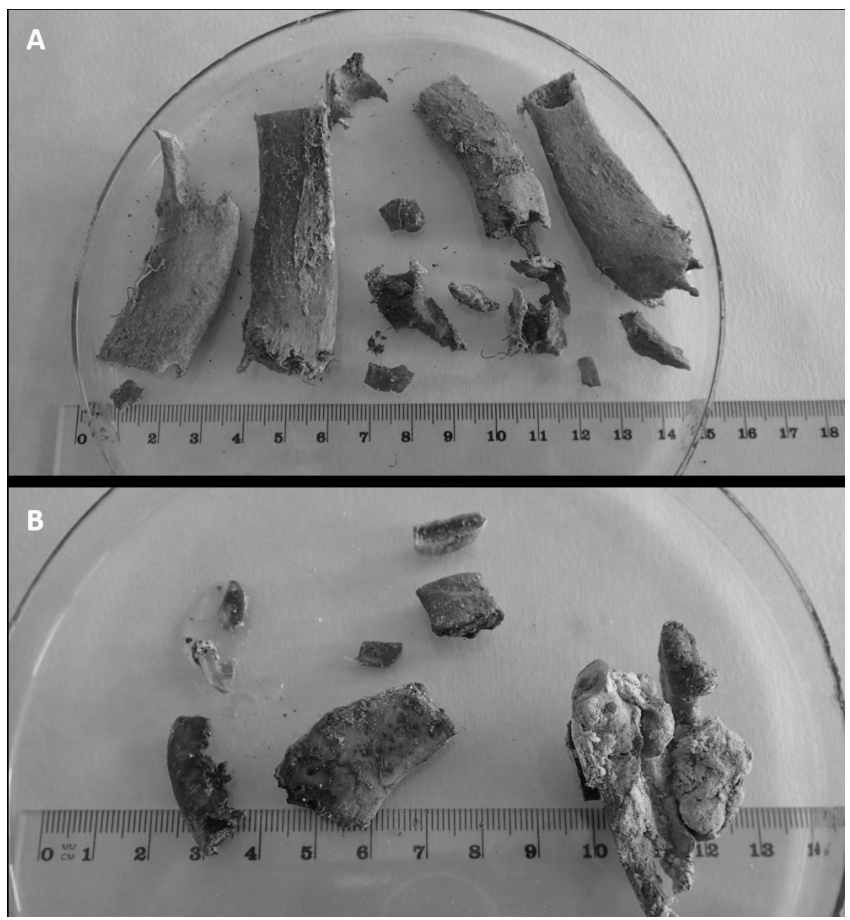

Figure 3. Morphological changes in costal cartilage in almost skeletonized cadavers: A - remains of costal cartilage collected from 67 -year-old male cadaver of unknown identity, found in the converted and abandoned bus 14 months after death; B - remains of costal cartilage collected from male cadaver of unknown identity and age, found in a ditch 3 years after death 
for $6 \mathrm{~h}$. The samples were additionally vortexed every hour to assure even penetration of the extraction mixture into the samples. Finally, the samples were centrifuged at 8,000 $\mathrm{rpm}$ for $30 \mathrm{sec}$ using a mini-spin centrifuge to remove the remaining residues.

\section{DNA Isolation and Analysis}

DNA isolation was performed using a Sherlock AX kit (A\&A Biotechnology, Poland). The quality and concentration of DNA samples were evaluated using a Quantifiler ${ }^{\mathrm{TM}}$ Trio DNA Quantification kit (Applied Biosystems, USA), HiD v. 1.2 software and Applied Biosystems ${ }^{\mathrm{TM}} 7500$ Real-Time PCR System (Applied Biosystems, USA). The final volume of the DNA solution used was $50 \mu$.

\section{Real-time PCR}

The qualitative analysis was performed using sequences for T. Large (214 bp) and T. Small (80 bp) autosomal chromosome as well as for chromosome $\mathrm{Y}$ (75 bp) (for male cadavers). Degradation index was calculated based on the ratio of $T$. Small to T. Large autosomal sequences. The PCR reaction was performed using a Power Plex ESX 17 and Power Plex HS 16 kit (Promega Corp., USA) in a Gene Amp PCR System 9700 thermocycler (Applied Biosystems, USA). Amplification products were separated toward DNA CC5 ILS 500 and CC5 ILS 600 standards (Promega Corp., USA) using 3130 Genetic Analyzer (Applied Biosystems, USA).

\section{Multiplex PCR}

Analyses of DNA profiles toward the following STR loci: AMEL, D3S1358, TH01, D21S11, D18S51, D10S1248, D1S1656, D2S1338, D16S539, D22S1045, VWA, D8SS1179, FGA, D2S441, D12S391, D19S433, SE33, D5S818, D13S317, D7S820, TPOX, CSF1PO, Penta D, and Penta E were carried out by identifying each locus according to international nomenclature using a Gene Mapper IDv. 3.2 software (Thermo Fisher Scientific, USA). Analyses of DNA profiles towards loci on the $Y$ chromosome were carried out with the use of the AmpFISTR $Y$ filer test (Applied Biosystems, USA). Also, forensic DNA typing was done using capillary electrophoresis.

\section{Statistical Analysis}

Distribution of variables was evaluated by the ShapiroWilk test and quantile-quantile plot. The interval data were expressed as a mean value \pm standard deviation, in the case of a normal distribution, or as a median (lower-upper quartiles), in the case of data with skewed or non-normal distribution. For comparison of data, the Kruskal-Wallis one-way analysis of variance was used. To determine the relationship between quantitative features, regression analysis was performed. Statistical significance was set at a $p$-value $<0.05$, and all tests were two-tailed. Statistical analysis was performed using Statistica 13.3 (TIBCO Software Inc.).

\section{RESULTS}

We determined the genetic profile of 79 cadavers because one sample was excluded from the analyses due to the sample condition and presence of water (Supplementary Table 1). The variety of dead body discovery places, and thus directly the decomposition conditions, is shown in Figure 4.

Due to the similar state of corpses, we pooled samples collected from cadavers discovered in flats $(n=16)$ and from patients after blood transfusion who died in the hospital ( $\mathrm{n}$ $=3$ ) into one group to facilitate statistical analyses. For the same reasons, we pooled the samples from exhumed dead bodies $(n=3)$ and samples from cadavers discovered in water $(n=4)$. We determined the genetic profile of 79 cadavers: 70 males and 9 females. Their average age at the time of death was $54.8 \pm 17.1$ years $(n=12)$. In 19 cases it was possible to determine PMI: the median time elapsed between the time of death and the time of body retrieval was 47 days (Q1, Q3 $-7,240$ days). These and other descriptive statistics of the examined group of cadavers and costal cartilage samples collected from them are shown in Supplementary Table 2.

Analyses showed that there are no statistically significant differences between $T$. Large and T. Small autosomal chromosomes ( $p=0.143$ and $p=0.146$, respectively) and chromosome $Y(p=0.132)$ sequences concentrations, as well as degradation index $(p=0.503)$ and the type of place in which the cadavers were found (Fig. 5-8; Table 1).

Also, there is no statistically significant difference between degradation index and PMI $(p=0.445)$ and between degradation index and cadaver's age $(p=0.677)$. These

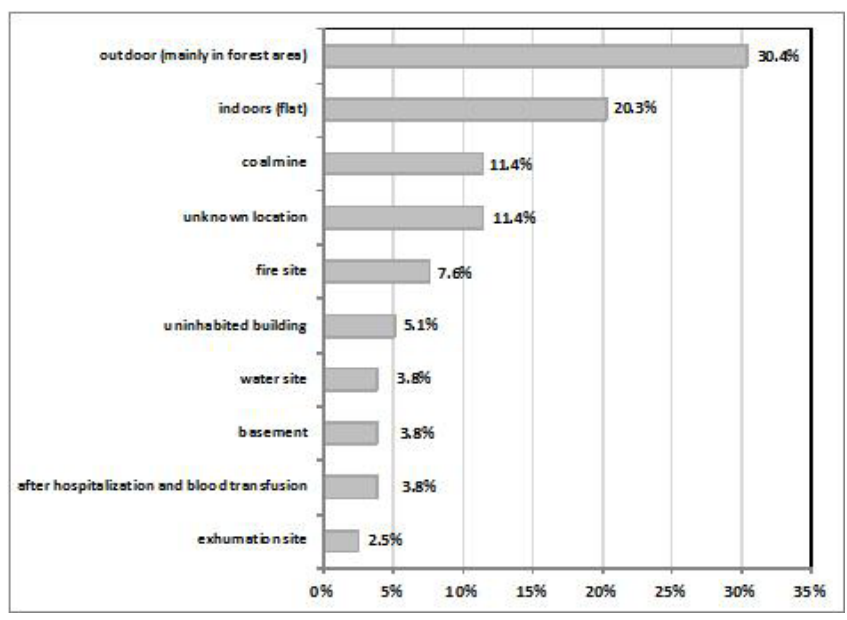

Figure 4. Places in which the cadavers included in the study were found 


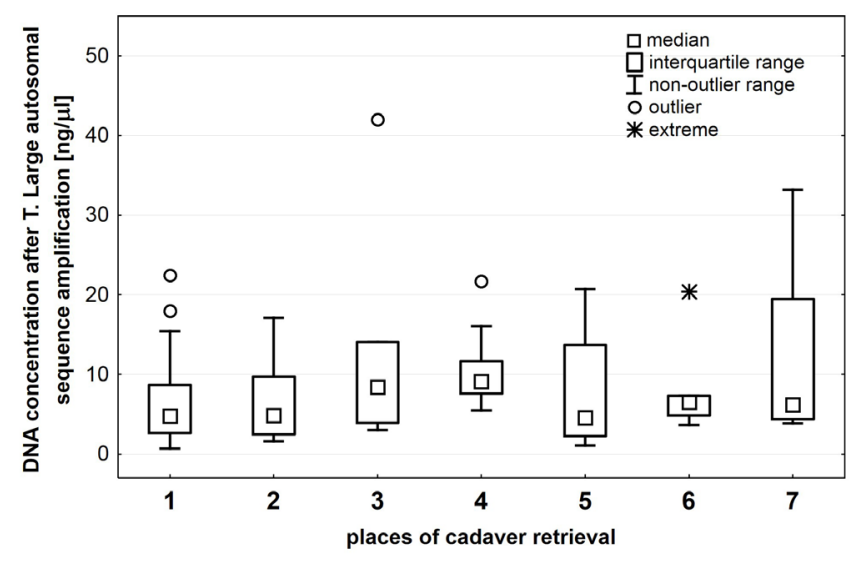

1 - indoors (flat/after hospitalization); 2 - outdoor (mainly in forest area); 3 - water/exhumed site; $\mathbf{4}$ - coal mine; 5 - fire site; $\mathbf{6}$ - basement/uninhabited building; 7 - unknown location

Figure 5. . DNA concentration [ng/ $\mu \mathrm{l}]$ after T. Large autosomal sequence (214 bp) amplification in costal cartilage samples collected from cadavers retrieved from different environments

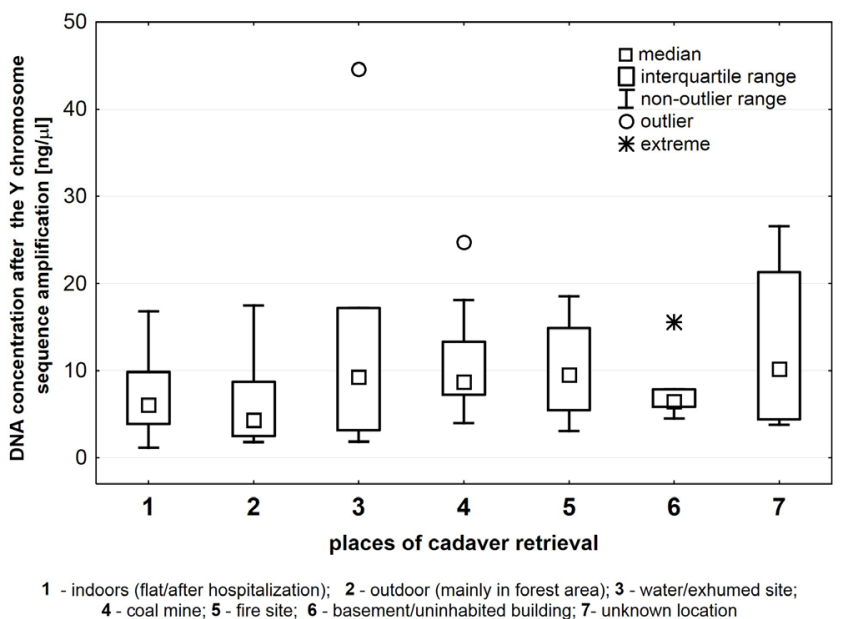

Figure 7. DNA concentration $[\mathrm{ng} / \mu \mathrm{l}]$ after the $\mathrm{Y}$ chromosome sequence $(75 \mathrm{bp})$ amplification in costal cartilage samples collected from male cadavers retrieved from different environments

results indicate that costal cartilage has a potential to be a suitable biological material for genetic individual tests of cadavers retrieved from different environments, regardless of the cadavers' age and time elapsed since death.

\section{DISCUSSION}

The current study aimed to prove that costal cartilage is a suitable forensic material for genetic individual identification purposes. Our results show that it is possible to extract a sufficient amount of DNA from costal cartilage to perform DNA tests, and the quality of the extracted DNA is good enough to successfully determine the cadaver's identity. The results show that it is possible to perform such tests regardless of

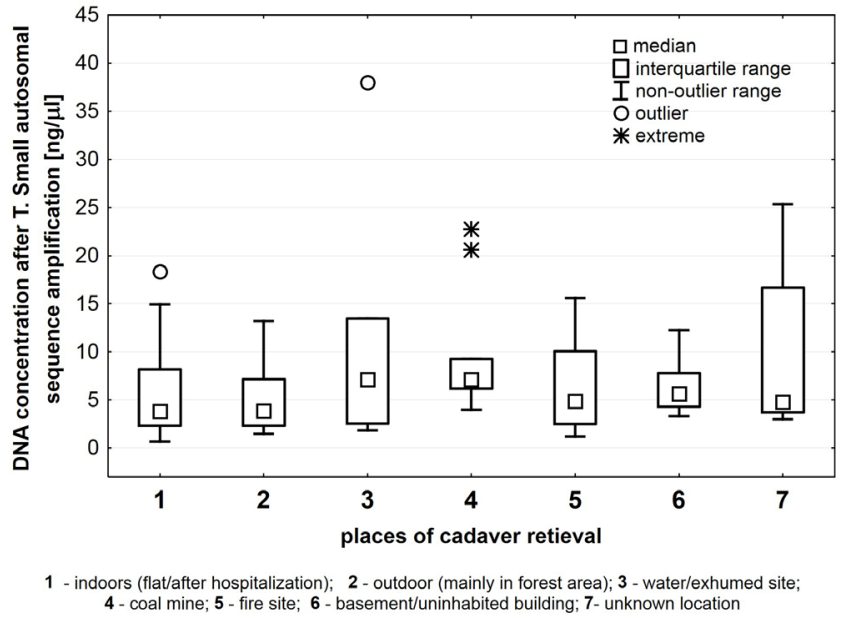

Figure 6. DNA concentration [ng/ $\mu \mathrm{l}]$ after T. Small autosomal sequence ( $80 \mathrm{bp}$ ) amplification in costal cartilage samples collected from cadavers retrieved from different environments

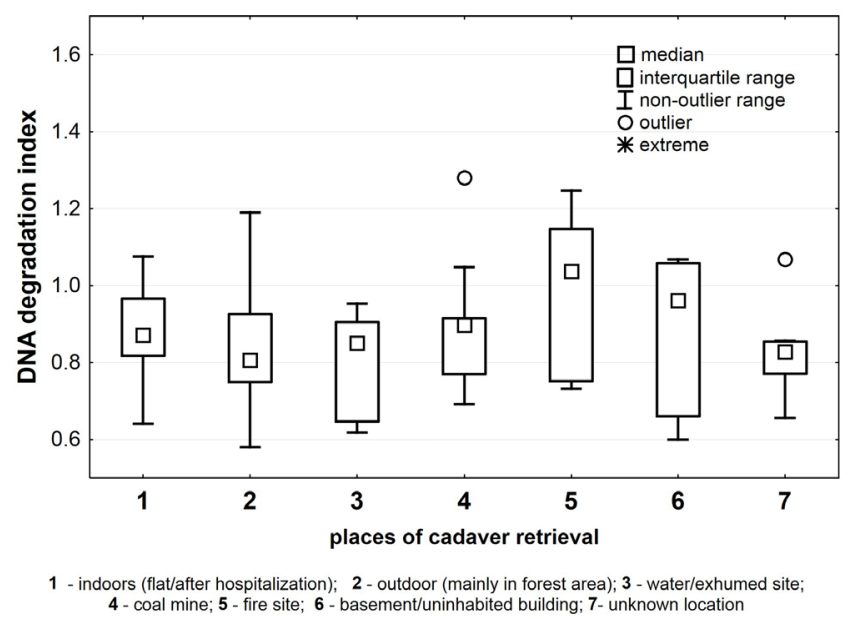

Figure 8. DNA degradation index* in costal cartilage samples collected from cadavers retrieved from different environments. ${ }^{*}$ T. Large amplicon DNA concentration/T. Small amplicon DNA concentration

the type of environmental factors that were affecting the decomposition process, the time elapsed between death and discovery, and the age of the deceased.

Forensic literature describes cases of successful use of different types of cartilage as a source of DNA for individual identification purposes based on STR analysis. Gino et al. reported three cases of individual identification performed with the use of epiglottis cartilage. The material was collected from the deceased exhumed 6,7 , and 18 months after death [16]. Siriboonpiputtana et al. described a case of successful DNA isolation from costal cartilage collected from the deceased retrieved from a river approximately three days after death [6]. Our results add to these findings, and show that it is possible to successfully extract good quality DNA 
from the costal cartilage regardless of the type of conditions affecting the decomposition process and the time elapsed between death and the cadaver's retrieval from the place of death. We extracted DNA from 79 cadavers retrieved from different conditions and at different times after death. We were able to successfully perform DNA typing for all samples - the DNA degradation index remained at the same level for cadavers retrieved from different environments (Table 1), and it was the same for cadavers with different PMls.

Zhouet et al. showed that DNA typing with costal cartilage as an investigative material gave $100 \%$ loci determination, compared to DNA typing with stains of blood, semen, saliva, or shredded epithelium as a DNA source [17]. Ferreira et al., while working on a carbonized car accident victim, compared the concentration of DNA isolated from different tissues; they proved that it is possible to extract more DNA from a hip joint cartilage than from a muscle [18]. On the other hand, in their previous work, Ferreira et al. extracted comparable concentrations of DNA from knee joint cartilage and phalanges of flood or mudslide casualties [19]. Our study showed that the costal cartilage is a good source of the DNA needed for individual identification tests. We were able to extract a comparable amount of DNA from almost all samples qualified for this study (failure rate of $1.3 \%$ ), and we were able to amplify all sequences for T. Large and T. Small autosomal chromosomes and chromosome $Y$ loci despite environmental factors, time elapsed, and cadaver's age, which could affect the decomposition process of the material.

Our results indicate that costal cartilage should be collected during forensic autopsies, as its degradation index is no different from samples collected from cadavers retrieved from different environments, and it does not change for samples collected from cadavers retrieved from the discovery sites at different PMls. This phenomenon may result from cartilage's unique structure. There are no blood vessels in this tissue; therefore, the chondrocytes surrounded by the extracellular matrix are less prone to degradation factors such as microorganisms [20]. Forensic literature lists other features that make cartilage a good biological material for genetic individual identification tests. The first of these is a low calcification degree, which simplifies DNA isolation by omitting the decalcification step [21, 22]. Next is tissue softness, which makes isolation of DNA from cartilage cheaper by not requiring the cryogenic mill needed for grinding hard tissues [17, 19, 23]. Finally, the last factor listed here is the fact that despite its mesodermal origin [24], the cartilage is not prone to the chimerism phenomenon that can occur after bone marrow transplantation; this excludes the possibility for giving a false opinion about the cadaver's identity [25]. 


\section{CONCLUSIONS}

We strongly suggest that costal cartilage should be listed as a biological material collected routinely during forensic autopsies, especially from cadavers in the advanced putrefaction process. We proved that it is possible to determine a full genetic profile from even small remains of cartilage collected from the region of rib bones' epiphysis. The cartilage structure protects chondrocytes' DNA from harmful environmental factors, even for extended periods of time. Moreover, unlike hard tissues, DNA isolation from costal cartilage is quicker, cheaper, and easier.

\section{Authors' Contributions}

M.T.: Research concept and design, supervising the project, carrying out the experiments, acquisition of data, drafting the article or revising it critically for important intellectual content, writing the manuscript; K.D.: Acquisition of data; G.T.J.: Final proofreading and approval of the version for publication; R.S.: Literature review; M.S.: Designing the figures; E.C.: Analysis and interpretation of data.

\section{Funding}

This work was performed at the Department of Forensic Medicine and Forensic Toxicology, Faculty of Medical Sciences in Katowice, Medical University of Silesia in Katowice, Poland and was supported by the institutional grant for young scientists of the Medical University of Silesia in Katowice (Poland) (grant no. KNW-2-O18/N/9/N and partially by grant no. KNW-1-162/N/9/Z, PCN-1-119/K/0/Z) and National Science Foundation (grant HRD 2011764).

\section{Conflict of Interest}

The authors have no potential conflicts of interest to declare.

\section{Ethics Approval}

Due to the nature of the research, the consent of the Ethics Committee was not required. The results were commissioned by the Public Prosecutor's Office. The Ethics Committee said it was not a medical experiment.

\section{Supplementary Material}

The Supplementary Tables 1 and 2 are available at Supplementary Materials.

\section{References}

[1] Sophia Fox A.J., Bedi A., Rodeo S.A.: The basic science of articular cartilage: Structure, composition, and function. Sports Health, 2009; 1: 461-468.

[2] Gentili C., Cancedda R.: Cartilage and bone extracellular matrix. Curr. Pharm. Des., 2009; 15: 1334-1348.

[3] Meier H., Springsklee M., Wildfeuer A.: Penetration of ampicillin and sulbactam into human costal cartilage. Infection, 1994; 22 : 152-155.

[4] Bolton S.N., Whitehead M.P., Dudhia J., Baldwin T.C., Sutton R.: Investigating the postmortem molecular biology of cartilage and its potential forensic applications. J. Forensic Sci., 2015; 60: 10611067.

[5] Meng H., Zhang M., Xiao B., Chen X., Yan J., Zhao Z., Ma K., Shen Y., Xie J.: Forensic age estimation based on the pigmentation in the costal cartilage from human mortal remains. Leg. Med., 2019; 40: 32-36.

[6] Siriboonpiputtana T., Rinthachai T., Shotivaranon J., Peonim V., Rerkamnuaychoke B.: Forensic genetic analysis of bone remain samples. Forensic Sci. Int., 2018; 284: 167-175

[7] Malaver P.C., Yunis J.J.: Different dental tissues as source of DNA for human identification in forensic cases. Croat. Med. J., 2003; 44: 306-309.

[8] Gaudio D., Fernandes D.M., Schmidt R., Cheronet O., Mazzarelli D., Mattia M., O'Keeffe T., Feeney R.N., Cattaneo C., Pinhasi R.: Genome-wide DNA from degraded petrous bones and the assessment of sex and probable geographic origins of forensics cases. Sci. Rep., 2019; 9: 8226.

[9] Vanek D., Budowle B., Dubska-Votrubova J., Ambers A., Frolik J., Pospisek M., Al Affefi A.A., Al Hosani K.I., Allen M., Al Naimi S.K., et al.: Results of a collaborative study on DNA identification of aged bone samples. Croat. Med. J., 2017; 58: 203-213.

[10] Rohland N., Glocke I., Aximu-Petri A., Meyer M.: Extraction of highly degraded DNA from ancient bones, teeth and sediments for high-throughput sequencing. Nat. Protoc., 2018; 13: 2447-2461.

[11] Edson S.M., McMahon T.P.: Extraction of DNA from skeletal remains. Methods Mol. Biol., 2016; 1420: 69-87.

[12] Ossowski A., Kuś M., Brzeziński P., Prüffer J., Piątek J., Zielińska G., Bykowska M., Jałowińska K., Torgaszev A., Skoryukov A., et al.: Example of human individual identification from World War II gravesite. Forensic Sci. Int., 2013; 233: 179-192.

[13] Damgaard P.B., Margaryan A., Schroeder H., Orlando L., Willerslev E., Allentoft M.E.: Improving access to endogenous DNA in ancient bones and teeth. Sci. Rep., 2015; 5: 11184. 
[14] Jakubowska J., Maciejewska A., Pawłowski R.: Comparison of three methods of DNA extraction from human bones with different degrees of degradation. Int. J. Legal Med., 2012; 126: 173-178.

[15] Emmons A.L., Davoren J., DeBruyn J.M., Mundorff A.Z.: Inter and intra-individual variation in skeletal DNA preservation in buried remains. Forensic Sci. Int. Genet., 2020; 44: 102193.

[16] Gino S., Robino C., Bonanno E., Torre C.: DNA typing from epiglottic cartilage of exhumed bodies. Int. Congr. Ser., 2003; 1239: 885-887.

[17] Zhou H., Wu D., Chen R., Xu Y., Xia Z., Guo Y., Zhang F., Zheng W.: Developmental validation of a forensic rapid DNA-STR kit: Expressmarker 16. Forensic Sci. Int. Genet., 2014; 11: 31-38.

[18] Ferreira S.T., Paula K.A., Nogueira R.F., Oliveira E.S., Moraes A.V.: A comparative study between muscle, cartilage and swab from inside the urinary bladder samples for DNA typing of severely burnt bodies in disaster victim identification (DVI). Forensic Sci. Int: Genet. Suppl. Series, 2015; 5: e617-e618.

[19] Ferreira S.T., Garrido R.G., Paula K.A., Nogueira R.C., Oliveira E.S., Moraes A.V. Cartilage and phalanges from hallux: Alternative sources of samples for DNA typing in disaster victim identification (DVI). A comparative study. Forensic Sci. Int: Genet. Suppl. Series, 2013; 4: e366-e367.
[20] Gao Y., Liu S., Huang J., Guo W., Chen J., Zhang L., Zhao B., Peng J., Wang A., Wang Y., et al.: The ECM-cell interaction of cartilage extracellular matrix on chondrocytes. Biomed. Res, Int., 2014; 2014: 648459.

[21] Forman J.L., Kent R.W.: The effect of calcification on the structural mechanics of the costal cartilage. Comput. Methods Biomech. Biomed. Engin., 2014; 17: 94-107.

[22] Sunwoo W.S., Choi H.G., Kim D.W., Jin H.R.: Characteristics of rib cartilage calcification in Asian patients. JAMA Facial Plast. Surg., 2014; 16: 102-106.

[23] Ossowski A., Diepenbroek M., Kupiec T., Bykowska-Witowska M., Zielińska G., Dembińska T., Ciechanowicz A.: Genetic identification of communist crimes' victims (1944-1956) based on the analysis of one of many mass graves discovered on the Powazki Military Cemetery in Warsaw, Poland. J. Forensic Sci., 2016; 61: 1450-1455.

[24] Aoyama H., Mizutani-Koseki S., Koseki H.: Three developmental compartments involved in rib formation. Int. J. Dev. Biol., 2005; 49: 325-333.

[25] Seo Y., Uchiyama D., Kuroki K., Kishida T.: STR and mitochondrial DNA SNP typing of a bone marrow transplant recipient after death in a fire. Leg. Med., 2012; 14: 331-335. 